\title{
Editorial
}

Pensar en Movimiento:

Revista de Ciencias del Ejercicio y la Salud

EISSN 1659-4436

Vol. 12, No.2, pp. 1- 9

\section{LA PUBLICACIÓN MULTILINGÜE COMO HERRAMIENTA LEGÍTIMA PARA \\ AUMENTAR EL ACCESO A LA CIENCIA (versión original en español) $^{1}$}

\author{
Luis Fernando Aragón Vargas, Ph.D.; FACSM \\ LUIS.ARAGON@ucr.ac.cr \\ Escuela de Educación Física y Deportes, Universidad de Costa Rica, Costa Rica
}

Manuscrito publicado: 12/12/2014

doi: http://dx.doi.org/10.15517/pensarmov.v12i2.17583

\begin{abstract}
RESUMEN
Aragón-Vargas, L.F. (2014). La publicación multilingüe como herramienta legítima para aumentar el acceso a la ciencia. PENSAR EN MOVIMIENTO: Revista de Ciencias del Ejercicio y la Salud, 12 (2), 1-9. La publicación redundante, que incluye el envío o la publicación del mismo manuscrito en dos o más idiomas, se considera una conducta científica inaceptable. Mientras tanto, la ejecución correcta de un proceso de publicación multilingüe ha sido una excepción difícil de manejar, en lugar de ser parte del proceso normal de publicación científica. Si bien es cierto que la publicación de un manuscrito en un segundo o tercer idioma no debería recibir el mismo crédito que la publicación original, tampoco debería ser castigada. Siempre y cuando se tomen los pasos necesarios y se hagan las declaraciones apropiadas, más bien debería ser algo que se promueva, pues se trata de una contribución que facilitará la comunicación científica y podría disminuir la brecha del conocimiento alrededor del mundo. En este documento se resumen las distintas formas de publicación multilingüe y se presentan algunas pautas básicas para la publicación válida del mismo manuscrito en distintos idiomas.
\end{abstract}

Palabras claves: publicación redundante, ética científica, ética editorial, traducción

Según el Comité Internacional de Editores de Revistas Médicas (International Committee of Medical Journal Editors-ICMJE), el envío de "el mismo manuscrito, en el mismo idioma o en idiomas distintos, en forma simultánea a varias revistas" se considera una conducta científica inaceptable (International Committee of Medical Journal Editors, 2014, Traducción libre). Existen buenas razones, muy sólidas, para esta política, sin embargo yo (inspirado por un editorial publicado por Zhang en 2013) quisiera presentar una justificación y algunas pautas obligatoriamente preliminares para los editores de

\footnotetext{
${ }^{1}$ También disponible en inglés y en portugués en esta misma revista
} 
revistas científicas, para la publicación legítima del mismo manuscrito en dos o más idiomas. Dicha práctica podría mejorar la comunicación científica y no debería considerarse una conducta científica inaceptable.

El argumento principal a favor de la publicación multilingüe es la necesidad de hacer que la ciencia de los países secundarios o periféricos sea más visible. Varias publicaciones se han referido a la ciencia escondida o "perdida": investigaciones valiosas en lenguajes secundarios como el español o el portugués, que generalmente son ignoradas en las revisiones sistemáticas y ni siquiera los científicos las leen (Gibbs, 1995; Stolerman \& Stenius, 2008; Williams, Bórquez, \& Basáñez, 2008). A pesar de ser el inglés el idioma principal para la comunicación científica, el conocimiento no debería tener un único idioma.

\section{Publicación bilingüe}

La publicación multilingüe de trabajos científicos asume distintas formas. Por ejemplo, el mismo manuscrito se podría publicar en dos o más idiomas en la misma revista, pero no existen pautas claras para hacerlo. Algunas revistas han publicado un único artículo (con una única identificación de objeto digital-DOI) en dos idiomas, lado a lado en el mismo archivo electrónico o en la misma copia impresa (cp. Jeukendrup, 2013). A eso se le puede llamar una publicación bilingüe, pero tiene limitaciones porque el lenguaje secundario no es visible para los motores de búsqueda o buscadores y el DOI conduce solamente a la información sobre el lenguaje primario. Debido a estas limitaciones, aquellos lectores que más se podrían beneficiar de la disponibilidad de la información en su propio idioma podrían no percatarse de su existencia. Además, aunque la citación del trabajo podría considerarse sencilla, no existe total transparencia, ya que el idioma utilizado para el envío y el proceso de revisión, que no necesariamente calza con el idioma primario de la revista, normalmente no se revela.

\section{Publicación multilingüe simultánea}

Otras revistas ponen a disposición el mismo artículo en dos o más idiomas simultáneamente, pero como archivos electrónicos separados, aunque utilizan el mismo DOI (cp. Angelo, Leite, \& Castilho, 2011), a lo cual le podemos llamar publicación multilingüe simultánea. El servicio es muy útil para quienes saben dónde buscar pero, una vez más, podría no ser visible para los motores de búsqueda. Es sorprendente que muchas revistas opten por asignar un único DOI a las versiones en distintos idiomas, pues los principales programas de gestión de revistas como Open Journals System (OJS) le asignan direcciones (URL) separadas a las versiones en distintos idiomas del mismo trabajo, y CrossRef ofrece una forma para usar un DOI distinto para cada idioma (CrossRef, 2014).

La publicación multilingüe simultánea tiene el potencial de cumplir un papel muy importante en la comunicación científica, como lo sugirió recientemente Salager-Meyer (2014). Actualmente, en reconocimiento de la calidad del trabajo que están realizando los científicos cuya primera lengua no es el inglés, muchas de las revistas principales ofrecen 
servicios profesionales de traducción (cobrados), para facilitarles la publicación en ese idioma. Cuando estos autores optan por usar estos servicios en lugar de utilizar su propio idioma están, en efecto, cerrándole las puertas a muchos de sus colegas que no tendrán acceso a la información. Esta restricción podría ser tan relevante como la que antes imponían las revistas de acceso por suscripción, una limitación que se está superando gracias al movimiento de Acceso Abierto, cuyas consecuencias incluyen embargos más cortos, depósito obligatorio en repositorios públicos y otros cambios en los modelos de publicación.

La publicación multilingüe simultánea es muy deseable porque va dirigida a la población que a menudo aportó los fondos y quizás a quienes participaron en las investigaciones. También coloca la información científica a la disposición inmediata de los científicos locales o regionales en su idioma natal, a la vez que satisface la necesidad de los autores de darle visibilidad a su trabajo en el mundo angloparlante.

Al optar por publicar el mismo manuscrito en dos o más idiomas, la revista ofrece un servicio de suma importancia, a la vez que evita la mayoría -aunque no la totalidadde los problemas que causan las publicaciones duplicadas, según han sido enumeradas por Alfonso y colaboradores (Alfonso, Bermejo, \& Segovia, 2005). Solamente se lleva a cabo un proceso de revisión, hay transparencia y la revista puede decidir si tiene el espacio y los recursos disponibles para hacerlo sin perjudicar los artículos convencionales. Además, la práctica actual puede mejorarse considerablemente de varias maneras: en primer lugar, tanto las publicaciones bilingües como las publicaciones multilingües simultáneas deberían incluir una declaración, probablemente junto a la fecha de envío del manuscrito, del idioma que se utilizó para el envío y la revisión. En segundo lugar, es necesario poner en práctica un mejor sistema que permita la citación independiente de la versión en cada idioma, puesto que siempre existirán diferencias que pueden crear confusión; se recomienda citar siempre la versión que de hecho leyeron los autores. En tercer lugar, para que las versiones en idiomas secundarios tengan visibilidad y sean ampliamente accesibles, se recomienda la indización independiente así como la asignación de una identificación de objeto digital distinta para cada una, según las instrucciones de CrossRef (2014). Finalmente, el hecho de que el trabajo esté disponible en otros idiomas debería declararse en forma explícita en la primera página del manuscrito y en los metadatos.

\section{Traducción de trabajos previamente publicados}

Otra modalidad es la traducción de un trabajo ya publicado a dos o más idiomas en revistas distintas, que para efectos de este artículo llamaré publicación multilingüe secuencial. El Comité Internacional de Editores de Revistas Médicas recomienda esta modalidad si el objetivo es diseminar información relevante dirigida a un máximo público posible (International Committee of Medical Journal Editors, 2014). Algunas revistas han traducido y publicado políticas o declaraciones de consenso de varias organizaciones de calibre mundial (cp. Alfonso et al., 2005), pero no es sino hasta ahora que se han publicado pautas para la publicación en idiomas secundarios (International Committee of 
Medical Journal Editors, 2014) o directrices para las traducciones al inglés (The European Association of Science Editors, 2014); estas últimas son más de carácter técnico y no se refieren explícitamente a la traducción de manuscritos publicados.

Algunas organizaciones como el Colegio de Medicina Deportiva de los EE.UU. (American College of Sports Medicine) han encargado la traducción oficial de sus declaraciones de consenso a distintos idiomas (ср. American College of Sports Medicine, 2014), pero los documentos que se producen no se publican en revistas científicas y generalmente son visibles solamente para los miembros de la organización o a quienes visitan su página en la Internet. Se consideran así un servicio para los usuarios, más que publicaciones. Si bien es cierto que la publicación oportuna de este tipo de trabajos sería muy deseable, sí debería existir un intervalo, acordado con antelación, entre la publicación original y la publicación secundaria (traducida), para darle prioridad a la primera (International Committee of Medical Journal Editors, 2014).

Generalmente no existe un equilibrio entre el trabajo intelectual involucrado en estas traducciones y el crédito otorgado, lo cual plantea una pregunta interesante sobre autoría: si una obra traducida (secundaria) se publica, ¿quiénes deberían ser los autores, los mismos de la obra original, o el científico que tradujo el manuscrito, con las debidas declaraciones y permisos? ¡La respuesta no es tan obvia como pareciera, pues algunos de los autores originales, o todos ellos, podrían estar reacios a asumir responsabilidad por la publicación de un manuscrito en un lenguaje que no comprenden! Otros asuntos que exigen discusión y aclaración incluyen la revisión del trabajo: el editor de la revista de la publicación secundaria podría considerar innecesario realizar una revisión a ciegas convencional por pares, pero podría decidir que es necesario contratar un profesional que certifique la precisión de la traducción. Estos y otros temas varían según el orden de la secuencia de publicación en los distintos idiomas.

La traducción de trabajos publicados en inglés a idiomas secundarios es bastante frecuente. Como ejemplo de las ciencias del ejercicio, la Editorial Biosystem de Argentina publicó versiones completas de trabajos originales de investigación, traducidas profesionalmente, desde 1997 o antes, en un esfuerzo por poner el trabajo original a disposición de los científicos y profesionales de habla hispana. Se anima a los autores y las editoriales de las obras originales en inglés a otorgar los permisos correspondientes para este tipo de esfuerzos, ya que la publicación en un idioma secundario alcanzará a una audiencia distinta, lo cual aumenta la diseminación del conocimiento y lo hace más universal, en línea con las recomendaciones de Salager-Meyer para mejorar la calidad de la ciencia en lo que él llama la periferia (Salager-Meyer, 2008).

Por otro lado, las revistas de corriente principal podrían estar dispuestas a considerar la publicación de trabajos publicados en otros idiomas, pero esto es poco probable. El modelo estará más propenso a ser juzgado como publicación redundante, y los autores tendrían que justificar por qué el envío no se hizo en inglés desde un principio. Esto es lamentable, pues mucha gente alrededor del mundo comparte la experiencia del profesor Gilson Volpato (una autoridad en publicación científica en Brasil), quien resalta el valor de la publicación multilingüe para los no científicos y los profesionales. Dice él: 
"descubrí acerca de algunos trabajos muy interesantes de investigadores japoneses, gracias a que un Israelita los tradujo al inglés en 1977" (comunicación personal, 2014). Independientemente de ello, bajo el modelo actual, ninguna revista de corriente principal querrá considerar una publicación secundaria, lo cual le abre una oportunidad importante a las revistas de la periferia que, además de aportar títulos, metadatos y resúmenes (abstracts) en inglés, deberían poner en práctica la publicación multilingüe simultánea de algunos manuscritos completos. Estas revistas podrían también considerar la posibilidad de traducir manuscritos ya publicados en su propio idioma, como publicaciones secundarias en inglés.

\section{Transparencia, cumplimiento y asuntos pendientes}

La publicación legítima de un manuscrito traducido no solo debería cumplir estrictamente con la ley de propiedad intelectual, sino que tendría que cumplir con criterios preestablecidos para efectos de transparencia, particularmente si se trata de una publicación secuencial. Entre ellos: obtener permisos por escrito, tanto de la editorial como de todos los autores del artículo original; revelar la naturaleza del artículo traducido desde el instante de su envío a una revista secundaria; incluir en el cuerpo del manuscrito una justificación de los autores para la traducción; resaltar la información relevante en el título del artículo y en sus metadatos; citar debidamente el artículo original (International Committee of Medical Journal Editors, 2014; Zhang, 2013).

Un asunto importante que está pendiente por resolver es el manejo del mérito académico: como no se trata de un trabajo original, una publicación secundaria (traducida) no debería contabilizarse, o debería tener una ponderación muy distinta (en realidad hace una contribución importante al conocimiento en el idioma secundario), cuando se realizan evaluaciones cuantitativas para instituciones o autores específicos (Zhang, 2013). Para esto se requiere de políticas y algoritmos específicos, pero los elementos de transparencia en el párrafo anterior deberían ser útiles para su puesta en práctica.

\section{Comentarios finales}

Se anima a los lectores a considerar este mismo editorial como ejemplo: si se publicara únicamente en inglés, los autores de idiomas secundarios no se enterarían fácilmente acerca de estas importantes tendencias que podrían tener un impacto directo sobre su trabajo. Por otra parte, si se publicara únicamente en español o en portugués, los editores de la mayoría de las revistas principales se mantendrían al margen de la discusión. Confiamos en que este editorial animará a más revistas periféricas a incluir la publicación multilingüe. Asimismo, invitamos a los autores interesados a traducir este editorial, con el debido respeto a su licencia de atribución, a otros idiomas secundarios.

La publicación multilingüe ha sido, hasta muy recientemente, una excepción difícil de manejar, en vez de ser parte del proceso normal de publicación científica. Si bien es cierto que la publicación de un manuscrito en un segundo o tercer idioma no debería recibir el mismo crédito que la publicación original, tampoco debería ser castigada. 
Siempre y cuando se tomen los pasos necesarios y se hagan las declaraciones apropiadas, más bien debería ser algo que se promueva, pues se trata de una contribución que facilitará la comunicación científica y podría disminuir la brecha del conocimiento alrededor del mundo.

En este documento se ha hecho un esfuerzo por clasificar los diferentes tipos de publicaciones multilingües (ver tabla 1) y se han presentado unas cuantas recomendaciones que deberían servir como documento de trabajo para la publicación posterior de las pautas necesarias.

$-6-$ 
Tabla 1.

Propuesta de clasificación para los procesos de publicación redundante en dos o más idiomas.

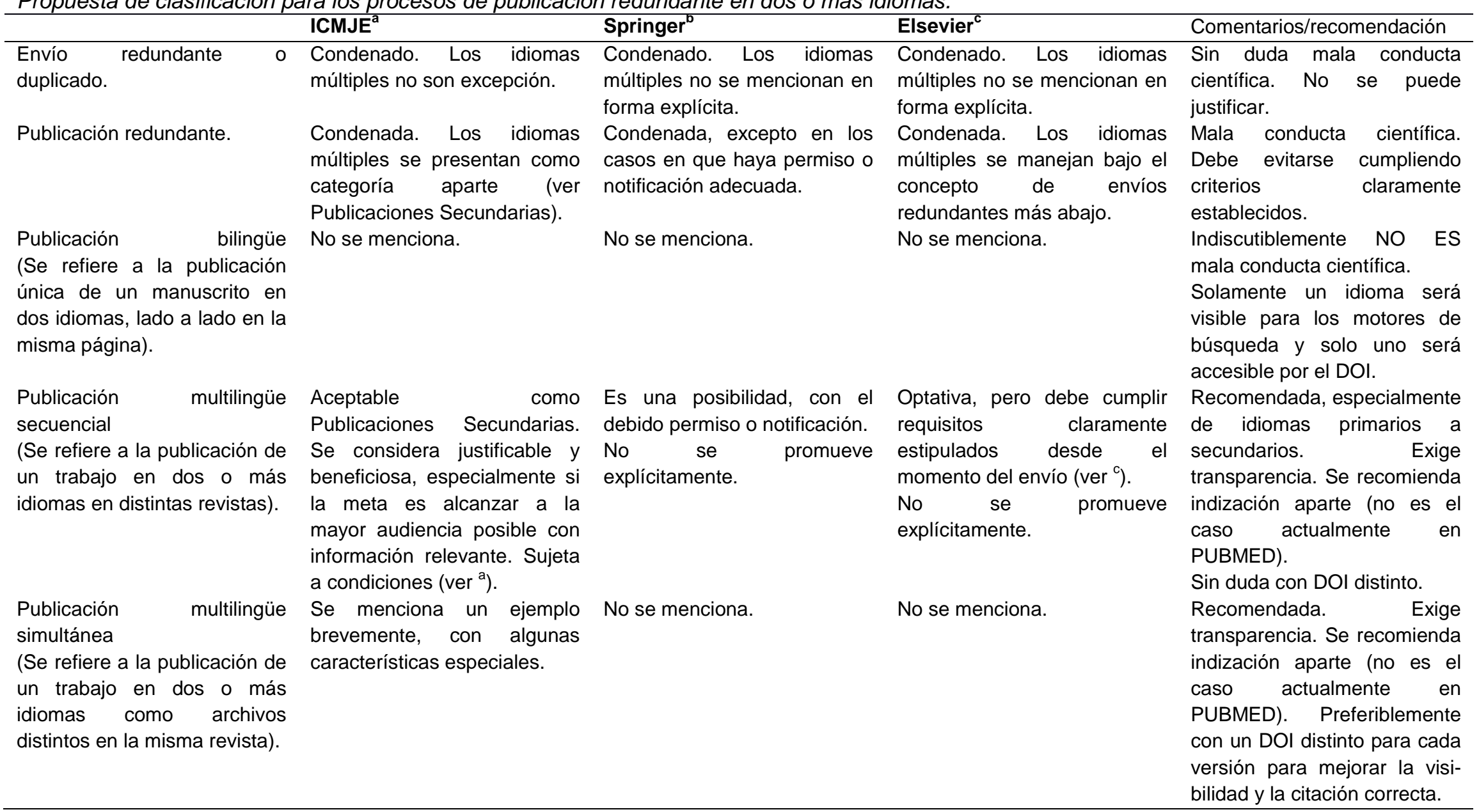

a Elaborado a partir de "Overlapping publications" por International Committee of Medical Journal Editors, 2014.

b Elaborado a partir de "Publishing ethics for journals. A guide for Editors-in-Chief, Associate and Managing editors" por Springer, $\underline{\text { n.d. }}$

C Elaborado a partir de "Identify 3. What identifies a case in this area?" por Elsevier, 2014. 


\section{Referencias}

American College Sports Medicine. (2014). Translated Position Stands. Recuperado de: http://www.acsm.org/about-acsm/international-outreach/translated-position-stands

Alfonso, F., Bermejo, J., \& Segovia, J. (May, 2005). Duplicate or Redundant Publication: Can We Afford It? Revista Española de Cardiología (English Edition), 58(5), 601604. doi: http://dx.doi.org/10.1016/S1885-5857(06)60739-1

Angelo, M., Leite, M. M. J., \& Castilho, V. (2011). Journal networking in nursing: a challenge to be shared. Revista Da Escola de Enfermagem Da USP, 45(6), 12791280. doi: http://dx.doi.org/10.1590/S0080-62342011000600001

CrossRef. (n.d.). Assigning DOIs to translations. Recuperado de: http://help.crossref.org/translations

Elsevier. (2014). Identify 3. What identifies a case in this area? Recuperado de: http://www.elsevier.com/editors/perk/multiple,-duplicate,-concurrentpublicationsimultaneous-submission/identify-3

Gibbs, W. (August, 1995). Lost Science in the Third World. Scientific American, 273, 9299. Recuperado de: http://www.scientificamerican.com/article/lost-science-in-thethird-world/

International Committee of Medical Journal Editors. (2014). Overlapping publications. Recuperado de: http://www.icmje.org/recommendations/browse/publishing-andeditorial-issues/overlapping-publications.html

Jeukendrup, A. (2013). Los carbohidratos durante el ejercicio: la investigación de los últimos 10 años. Nuevas recomendaciones. Apunts, (113), 7-22. doi: http://dx.doi.org/10.5672/apunts.2014-0983.es.(2013/3).113.00

Salager-Meyer, F. (2008). Scientific publishing in developing countries: Challenges for the future. Journal of English for Academic Purposes, 7(2), 121-132. doi: http://dx.doi.org/10.1016/j.jeap.2008.03.009

Salager-Meyer, F. (2014). Writing and publishing in peripheral scholarly journals: How to enhance the global influence of multilingual scholars? Journal of English for Academic Purposes, 13, 78-82. doi: http://dx.doi.org/10.1016/j.jeap.2013.11.003

Stolerman, I. P., \& Stenius, K. (January, 2008). The language barrier and institutional provincialism in science. Drug and Alcohol Dependence, 92(1-2), 1-2. doi: http://dx.doi.org/10.1016/j.drugalcdep.2007.07.010

Springer. (n.d.). Publishing Ethics for Journals. A guide for Editors-in-Chief, Associate and Managing editors. Recuperado de: http://www.springer.com/gp/authorseditors/editors\#c4176 
The European Association of Science Editors. (2014). EASE Guidelines for Authors and Translators of Scientific Articles to be Published in English. Recuperado de: http://www.ease.org.uk/publications/author-guidelines

Williams, J. R., Bórquez, A., \& Basáñez, M.-G. (2008). Hispanic Latin America, Spain and the Spanish-speaking Caribbean: A rich source of reference material for public health, epidemiology and tropical medicine. Emerging Themes in Epidemiology, 5(1), 17. doi: http://dx.doi.org/10.1186/1742-7622-5-17

Zhang, Y. (2013). Bilingual (multilingual) publications and duplicate publications: for or against? Journal of Zhejiang University-SCIENCE A, 14(9), 687-690. doi: http://dx.doi.org/10.1631/izus.A1300272 